\title{
MODELO DE IMPACTO ECONÓMICO (MIE) ENFOCADO A SITUACIONES DE RACHAS MÁXIMAS EXTREMAS
}

\author{
M. Rodrigo ${ }^{(1)(2)(3)}$, J.A. López ${ }^{(2)}$ \\ (1) Consorcio de Compensación de Seguros (CCS), Paseo de la Castellana, 32, 28046, Madrid (España), \\ ccseguros@aemet.es

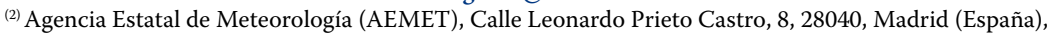 \\ jlopezd@aemet.es \\ (3) Aplicaciones y Tratamientos de Sistemas (ATSistemas), Calle Valle de Alcudia, 3, Edif.2, Planta 1, 28232, \\ Madrid (España), mrodrigo@atsistemas.com
}

\section{Resumen}

El Consorcio de Compensación de Seguros (CCS) tiene por objeto indemnizar, en la forma establecida en su Estatuto Legal y en régimen de compensación, las pérdidas derivadas de acontecimientos extraordinarios acaecidos en España. Dentro de estos acontecimientos se encuentran fenómenos de la naturaleza como la Tempestad Ciclónica Atípica (TCA), en la que están incluidos los vientos extraordinarios, definidos como aquellos que presentan rachas que superen los $120 \mathrm{~km} / \mathrm{h}$. Se entiende por racha el mayor valor de la velocidad del viento sostenida durante un intervalo de tres segundos.

El CCS solicita la colaboración a la Agencia Estatal de Meteorología (AEMET) en la delimitación geográfica del área de afectación del fenómeno meteorológico descrito mediante la extrapolación, con los criterios científicos más avanzados, de las mediciones existentes, de forma que se procure la mayor homogeneidad posible en la definición del área y se evite la exclusión de puntos aislados, teniendo en consideración las mediciones en los municipios limítrofes y, en su caso, los colindantes a estos (Consorcio de Compensación de Seguros, 2017).

La técnica geoestadística utilizada por AEMET para estimar las áreas en las que se producen estos vientos extraordinarios es el krigeado universal que se apoya en los datos de observación de racha máxima y toma como variables externas la elevación del terreno, la distancia al mar y las salidas del modelo de predicción HIRLAM (High Resolution Limited Area Model) del campo de racha máxima (Cressie, 1991).

En este trabajo se presenta el Modelo de Impacto Económico (MIE) desarrollado para las situaciones de rachas máximas extremas y para el área concreta de la España peninsular e islas Baleares. El objetivo fundamental de este modelo consiste en proporcionar, de una manera rápida, información significativa para la cobertura del riesgo. La evaluación de cada situación permitirá obtener, entre otros productos, una estimación inicial del coste de las pérdidas esperadas por vientos extremos tanto a nivel municipal como de manera global. Las estimaciones vienen dadas para cada municipio ya que esta es la unidad aplicada por el CCS en la cobertura de daños, esto es, la zona afectada se extiende a todo el municipio siempre que exista algún punto en el que se cumpla la condición de TCA (Rodrigo, M. y López, J.A., 2017). 


\section{Metodología y desarrollo del modelo}

El ajuste del Modelo de Impacto Económico utiliza las situaciones de racha máxima extrema ocurridas entre los años 2012 y 2015, ambos incluidos, así como una base de datos de catastro a nivel municipal, generada explícitamente para este fin. Por otro lado, surge la necesidad de caracterizar las situaciones con vientos extremos puesto que estas poseen características y extensiones muy diferentes.

El ajuste del modelo tiene en cuenta las situaciones de viento extremo ocurridas a partir del año 2012. Con el objetivo de homogeneizar el estudio no se ha considerado la información anterior a este año ya que el Reglamento de Seguro de Riesgos Extraordinarios modifica la definición de TCA tomando un límite diferente para las rachas máximas. En años anteriores al 27 de octubre de 2011 el límite impuesto en el valor de racha máxima para las situaciones de TCA era de $135 \mathrm{~km} / \mathrm{h}$, pasando luego al valor actual de $120 \mathrm{~km} / \mathrm{h}$. Además, en el primer período en el que el límite es de $135 \mathrm{~km} / \mathrm{h}$, no se incorporaba el modelo Hirlam en la interpolación (Rodrigo, M. y López, J.A., 2016b).

La base de datos catastral a nivel municipal, generada explícitamente para este fin, incluye los valores de construcción (tipo urbano y rústico), así como los valores tipo BICE (Bienes Inmuebles de Características Especiales) obtenidos a través de la Dirección General del Catastro. Los casos especiales de País Vasco y Navarra presentan valores estimados calculados en trabajos anteriores (Rodrigo, M. y López, J.A., 2016a). Se añadió información relativa a los vehículos, tomada de la Dirección General de Tráfico, entre otras fuentes, y valores de la exposición de la red ferroviaria de Adif, información aportada por el CCS. El conjunto final de los valores tiene en cuenta los términos aplicables en el campo de los seguros (franquicia, infraseguro y penetración).

Los daños totales producidos en una determinada TCA dependen de las características meteorológicas de la propia situación por lo que se desarrollaron diferentes índices de caracterización (máximo, de excesos y de excesos cuadrático) calculados para cada municipio y para dos variables diferentes: racha máxima del viento y velocidad máxima del viento promediada en 10 minutos, esta última también se considera importante para la caracterización de las situaciones en términos de su impacto en daños.

Tras las pruebas realizadas con estos diversos índices se eligió el índice de la ecuación (1), que se calcula para cada municipio y que utiliza un umbral para el valor de racha máxima de $70 \mathrm{~km} / \mathrm{h}$ relacionado, entre otros aspectos, con el límite impuesto por las compañías de seguros para aplicar la cobertura de los daños producidos. Este índice de excesos es el parámetro que caracteriza físicamente la intensidad de la TCA:

$$
\left.I_{\text {exc }}\right|_{\text {mиni }}=\frac{\sum_{i \in \text { max }}\left(0, R_{\text {max }}^{i}-70\right)}{n^{o} \text { ptos } \text { grid }\left.\right|_{\text {mииi }}} 70
$$

El Modelo de Impacto Económico construido, mediante el estudio de la correlación de diferentes variables, contiene un término dependiente del catastro y otro término que surge de la interacción del catastro con el índice de excesos de racha máxima con el umbral de $70 \mathrm{~km} / \mathrm{h}$, ambos términos participan en el modelo a nivel municipal. El modelo, además, añade un término global referido al catastro que modula las estimaciones finales. 
Otros detalles técnicos para el desarrollo del MIE son:

- Tal y como se expuso el límite dado por la definición de TCA es de 120 km/h.

- Los valores de observación utilizados engloban las estaciones pertenecientes a AEMET y a los Servicios Meteorológicos Externos de: MeteoGalicia, Euskalmet, MeteoNavarra, Gobierno de La Rioja y MeteoCat.

- La técnica de interpolación utilizada es el krigeado universal.

- El procedimiento seguido operativamente en AEMET utiliza el máximo valor de observación de racha máxima del período completo de días que comprenda la situación para estimar las velocidades de racha máxima en una única interpolación. En cambio, en la implementación actual del MIE, se realiza una estimación mediante krigeado universal para cada día que conforma la TCA para obtener, posteriormente, el máximo valor de las estimaciones en un único mapa, que es el producto final.

- Para conseguir una mayor homogeneidad y automatización, los parámetros establecidos para el interpolador y el semivariograma son fijos.

- La información aportada por el CCS tiene una gran relevancia para el ajuste por lo que se depura minuciosamente la información recibida. Principalmente se busca la compatibilidad en fechas (entre la base de datos de las indemnizaciones aplicadas por el CCS con el registro de TCA aportado por AEMET) y se eliminan los valores relativos a tornados y extensión de municipios al cubrir los daños.

- La técnica del krigeado universal empleada para cada una de las situaciones se aplica de manera automática utilizando R, lenguaje y entorno de programación con un enfoque al análisis estadístico ( $R$ Core Team, 2015), que utiliza la función krige para extraer tanto las medidas centrales como las de dispersión (esta última información ha sido aprovechada para realizar otros trabajos).

Además, en este caso, se elabora un estudio que compara estos resultados con los obtenidos mediante el paquete RSAGA de R que accede directamente al Sistema de Información Geográfica SAGA (Sistema para Análisis Automatizados Geocientíficos) que es la herramienta empleada operativamente y, por tanto, los resultados que mostraremos en este trabajo (Bivand, R.S. et al, 2008).

Los resultados obtenidos mediante el MIE vienen dados como el logaritmo de la indemnización en miles de euros, aportando un valor para cada municipio afectado por TCA así como un valor global de dicha situación. En ocasiones, cuando la situación de viento extremo presenta características de gran relevancia, con vientos generalizados muy elevados o extensiones amplias, el CCS extiende la cobertura del daño de manera que los municipios colindantes a los inicialmente afectados (anillo 1) y aquellos limítrofes a este primer anillo con rachas superiores a $100 \mathrm{~km} / \mathrm{h}$ (anillo 2) también quedan englobados en la TCA. El modelo, por tanto, considera este aspecto y calcula, para estos casos concretos, las estimaciones de las pérdidas derivadas con estas condiciones y teniendo en cuenta dos escenarios: aquel que no modifica las estimaciones de las pérdidas en niveles previos al añadir otros (denominado "CONS" de "conservador") y aquel que sí las modifica (denominado "NEW" de "nuevo"), este segundo escenario presentará valores estimados superiores al primero. 


\section{Resultados y discusión}

El MIE ha sido validado en situaciones posteriores al periodo utilizado en el ajuste, ocurridas a partir del año 2016, así como en las tempestades Gong y Dirk (ocurridas durante el año 2013 y que alcanzaron especial importancia tanto por su gran extensión como por su impacto económico). Para ello los valores de pérdidas estimados con el modelo se comparan con los valores cubiertos por el CCS en cada una de las situaciones.

En la figura 1 se muestran los resultados de las validaciones para las situaciones ocurridas a partir de 2016 y hasta abril de 2017, incluyendo las TCA Gong y Dirk (2013). En todas ellas se muestran las pérdidas estimadas teniendo en cuenta los dos anillos y

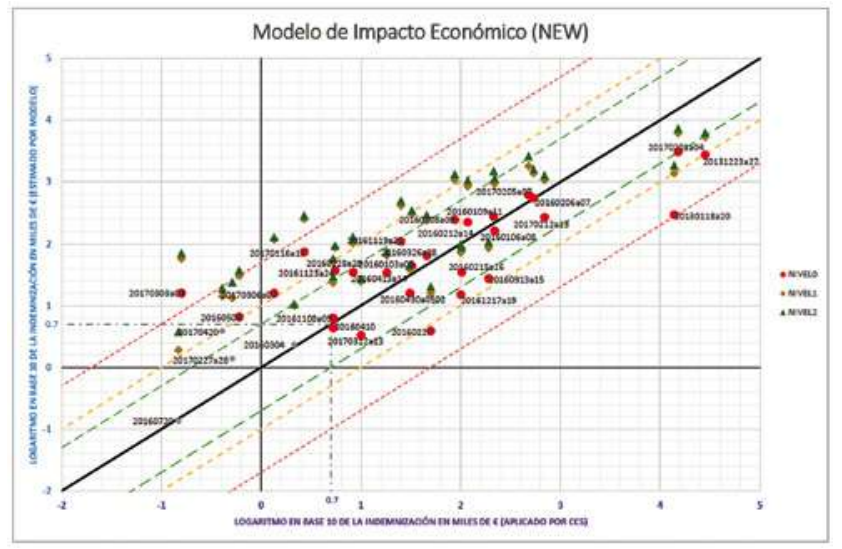

para el escenario que da valores más altos en las estimaciones, aquel que modifica las estimaciones de las pérdidas en niveles previos al añadir otros (escenario "NEW").

Fig. 1.- Pérdidas estimadas mediante MIE (eje de ordenadas) frente a pérdidas aplicadas por el CCS (eje de abscisas). Escenario « NEW ».

La figura 2 es similar a la figura 1 pero para el escenario que no modifica las estimaciones de las pérdidas en niveles previos al añadir otros (escenario "CONS").

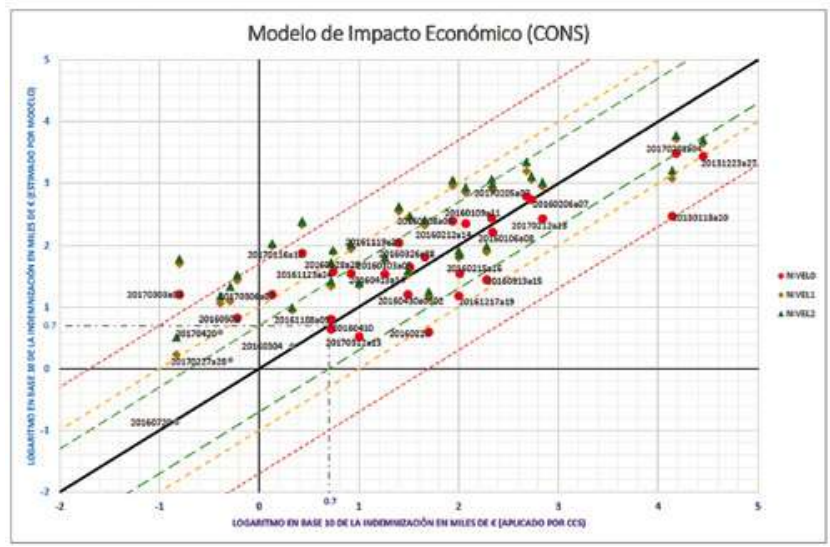

Fig. 2.- Pérdidas estimadas mediante MIE (eje de ordenadas) frente a pérdidas aplicadas por el CCS (eje de abscisas). Escenario «CONS». 
En las gráficas anteriores el nivel 0 se refiere a la estimación inicial del MIE que incluye los municipios afectados por TCA según el procedimiento operativo en AEMET (krigeado universal). El nivel 1 sería aquel que engloba los municipios afectados anteriormente y los municipios pertenecientes al anillo 1 , mientras que el nivel 2 da lugar a la máxima cobertura de municipios pues añade el anillo 2 (cuyas condiciones fueron ya señaladas).

Se aprecia en las figuras que los puntos forman una nube de puntos más o menos cercana a la diagonal principal (línea negra continua) que representaría un modelo ideal en el que la estimación de las pérdidas estimadas mediante MIE coincide exactamente con las indemnizaciones aplicadas por el CCS. Se han señalado en gris situaciones poco relevantes con valores demasiado bajos en las indemnizaciones (tanto estimadas como aplicadas).

Existen situaciones de TCA en las que el modelo sobreestima las pérdidas, cabe destacar que gran parte de ellas corresponden a fechas más cercanas (año 2017) y se debe a que los valores aplicados del CCS pueden estar subestimados a su vez, ya que el cierre de los expedientes indemnizados por parte del CCS tarda tiempo en realizarse debido a las gestiones que supone. Estos valores, por tanto, no habrían sido tenidos en cuenta en el momento de realizar el trabajo y dichas TCA pueden, realmente, tener una estimación mejor que la presentada aquí.

Por otro lado, los casos en que el MIE subestima las pérdidas corresponden principalmente a TCA de mayor relevancia, tanto por su extensión como por la pérdida económica. Estos casos se sitúan en el lado derecho de las gráficas. Cabe comprobar que el añadir anillos (que es el procedimiento habitual que sigue el CCS para la cobertura del riesgo) mejora apreciablemente las estimaciones.

De manera general pueden existir situaciones en las que se infraestiman o sobreestiman las pérdidas y que no siguen un patrón claro o una explicación demasiado lógica a simple vista puesto que las variables y factores que entran en juego en este estudio son demasiado dispersos y poco homogéneos. Trabajos posteriores se están centrando en ver cómo influye la longitud del número de días que comprende la situación en el momento de aplicar MIE y ver si esta vía podría mejorar el Modelo de Impacto Económico desarrollado hasta el momento.

El MIE está actualmente operativo y las estimaciones de las pérdidas ocasionadas por las situaciones de TCA son enviadas al CCS. La información enviada consiste, principalmente, en una estimación inicial de la pérdida global de la situación indicando el número de municipios y provincias afectados. Además, se detallan dichos municipios indicando un valor de pérdida estimado para cada uno de ellos. En la mayoría de los casos es suficiente englobar los municipios iniciales afectados por TCA (nivel 0), pero si se considera que la situación puede llegar a ser importante se añaden aquellos municipios pertenecientes a los niveles superiores dando ambos escenarios en las estimaciones, escenario "CONS" y escenario "NEW".

A modo de ejemplo se comenta una situación de gran relevancia, Ana, ocurrida del 10 al 11 de diciembre de 2017. En la figura 3 se detallan los municipios afectados en los 
diferentes niveles de acuerdo a una primera estimación provisional (la que es enviada inicialmente al CCS). El color rojo se refiere al nivel 0 y los niveles adicionales se corresponden con el amarillo (anillo 1) y el verde (anillo 2).

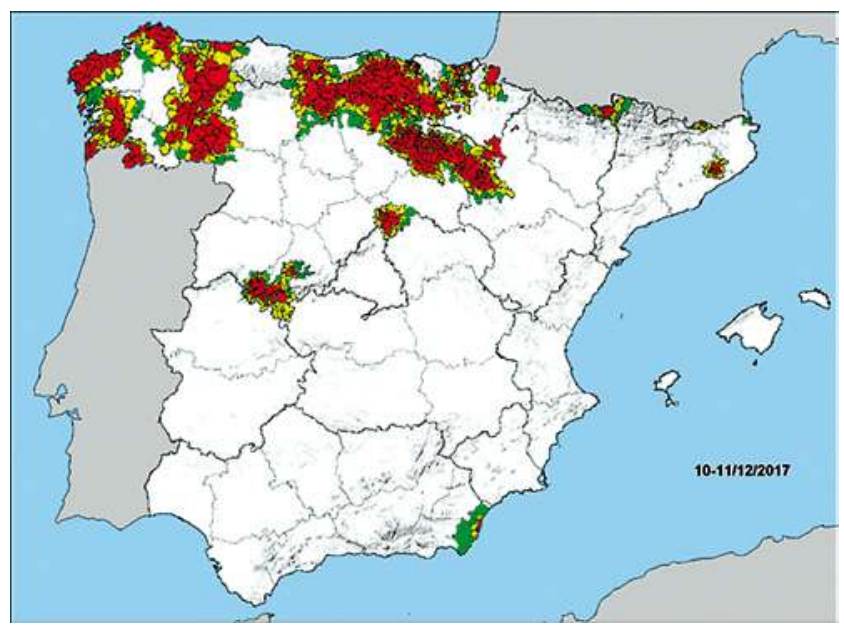

Fig. 3.- TCA Ana, 10 al 11 de diciembre de 2017. Municipios afectados hasta el nivel 2 .

En las figuras que se muestran a continuación reflejamos las estimaciones de las pérdidas obtenidas mediante MIE para la TCA señalada, Ana del 10 al 11 de diciembre de 2017, hasta el nivel más elevado (nivel 2) y para los dos escenarios desarrollados según el modelo: escenario "CONS", en figura 4, y escenario "NEW" en figura 5.

Es importante señalar que estos productos deben ser interpretados como las estimaciones iniciales y provisionales enviadas al CCS, los cuales sirven para tener una estimación previa al informe definitivo y final de AEMET. El objetivo es proporcionar, de una manera rápida, información significativa para la cobertura del riesgo pero esta

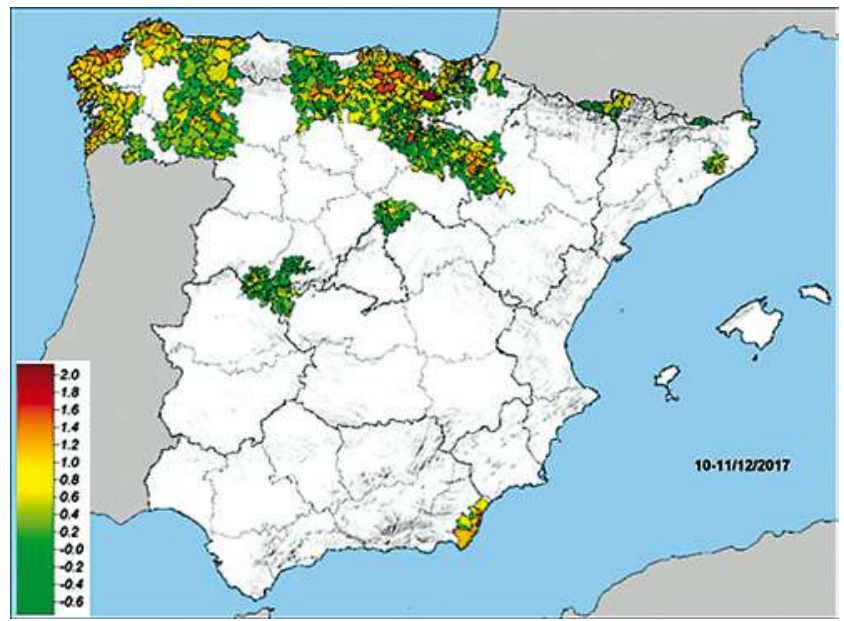

Fig. 4.- TCA Ana, 10 al 11 de diciembre de 2017. Estimaciones provisionales con MIE, incluye hasta el nivel 2 para el escenario « CONS». Los valores indicados se refieren al logaritmo decimal de la pérdida estimada en miles de euros. 
rapidez conlleva, en algunos casos, diferencias importantes con las estimaciones finales y definitivas. En general, en la mayoría de las ocasiones, supondrá una infraestimación en los propios valores de racha máxima. Un factor adicional de incertidumbre en las estimaciones proviene de que los datos de observación obtenidos tras los primeros días del evento TCA pueden no estar disponibles al no haber sido validados todavía. De hecho, es habitual que sean los valores más elevados de racha máxima, precisamente, los que no están disponibles de manera inmediata ya que son los que suelen presentar mayores problemas en la validación, y no contar con ellos desde un primer momento hace lógicamente que las estimaciones obtenidas sean menores que las derivadas en un tiempo posterior.

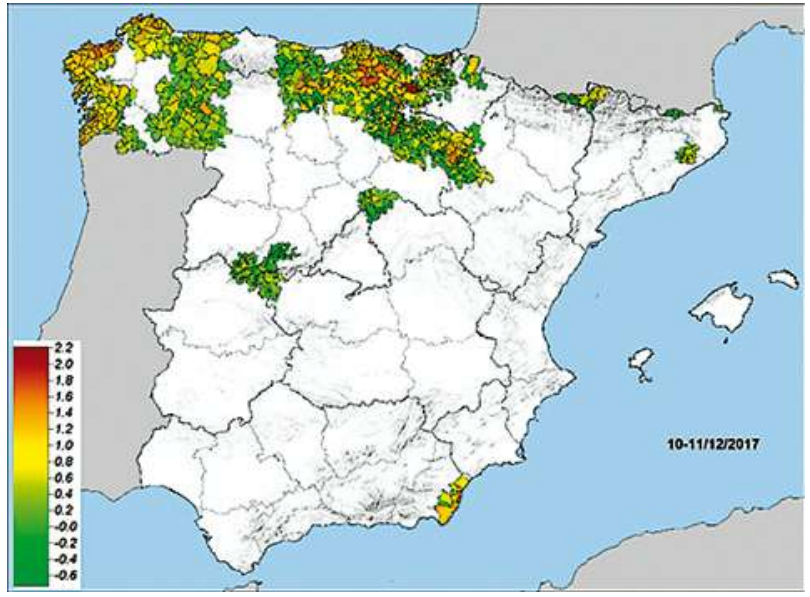

Fig. 5.- TCA Ana, 10 al 11 de diciembre de 2017. Estimaciones provisionales con MIE, incluye hasta el nivel 2 para el escenario « NEW ». Los valores indicados se refieren al logaritmo decimal de la pérdida estimada en miles de euros.

\section{Referencias}

- Bivand, R.S., Pebesma, E.J. y Gómez-Rubio, V. (2008). Applied Spatial Data Analysis with R. Editorial Springer.

- Consorcio de Compensación de Seguros, 2017: Recopilación Legislativa, Edición de febrero de 2017, 308 pp.

- Cressie, N., 1991. Statistics for spatial data. Editorial: A Wiley - Interscience Publication.

- R Core Team, 2015. R: A language and environment for statistical computing. R Foundation for Statistical Computing, Vienna, Austria. https://www.R-project.org/.

- Rodrigo, M. y López, J.A., 2016a. Modelo de impacto económico para las situaciones de rachas máximas extremas en la España peninsular. Publicaciones de la Asociación Española de Climatología (AEC), Serie A, no 10, pp. 323-333, ISBN 978-84-16724-19-2.

- Rodrigo, M. y López, J.A., 2017. Propuesta de modelos de impacto económico para situaciones de rachas máximas extremas. Desarrollo y aplicación. $10^{\circ}$ Simpósio de Meteorologia e Geof ísica da Associaçao Portuguesa de Meteorologia e Geofísica (APMG).

- Rodrigo, M. y López, J.A., 2016b. Resultados preliminares del modelo de impacto económico para las situaciones de rachas máximas extremas en la España peninsular. XXXIV Jornadas Científicas de la Asociación Meteorológica Española (AME). ISBN 978-84-617-5240-9 